\title{
Valores Morales y Familia en Estudiantes de Enseñanza Media de la Provincia de Concepción*
}

\section{Moral Values and Family in Middle School Students of the Concepción Province.}

\author{
M.E. Mathiesen, ${ }^{1}$ \\ O. Mora, ${ }^{2}$ I. Chambläs, ${ }^{3}$ G. Navarro, ${ }^{4}$ M. Castro. ${ }^{5}$
}

\begin{abstract}
Resumen
Se presenta un aspecto de la moralidad juvenil, medido por la aceptación de conductas morales cuestionables (Escala de Harding y Phillips, 1986), y sus relaciones con variables familiares, en una muestra aleatoria de 296 estudiantes de enseñanza media, de la provincia de Concepción, Chile.

El funcionamiento psicométrico de la escala de Harding y Phillips fue adecuado.

En general, la permisividad moral fue baja, con una más alta permisividad sexual y una más baja legal y personal.

Hubo correlación entre las variables funcionamiento familiar y los niveles de permisividad. A mayor familismo e interacción familiar, menor permisividad moral y a mayor insatisfacción con el funcionamiento familiar, mayor permisividad moral.
\end{abstract}

\footnotetext{
Abstract

This study presents an aspect of youth morality, especifically acceptance of questionable moral behaviors and its relation to familiy structure and functioning in students of secundary education at the Province of Concepción, Chile. For this purpose a random sample of 296 students was surveyed.

The Harding and Phillips Scale (1986) showed adequate psicometrical functioning.

In general, the moral permissiveness detected may be considered low, but higher levels of permissivenes were observed in sexuality dimention and lower levels were measured on personal and legal permissiveness.

* Esta investigación ha recibido financiamiento de la Dirección de Investigación de la Universidad de Concepción,

1 Antropóloga, Facultad de Ciencias Sociales, Universidad de Concepción. Chile.E-mail:mmathies@udec.cl

2 Asistente Social, Facultad de Ciencias Sociales, Universidad de Concepción. Chile.

3 Asistente Social, Facultad de Ciencias Sociales, Universidad de Concepción. Chile.

4 Psicóloga, Facultad de Ciencias Sociales, Universidad de Concepción. Chile.

5 Sociólogo, Facultad de Ciencias Sociales, Universidad de Concepción. Chile.
} Chile. 
A correlation was found between family and permissiveness. The greater familism and family interaction in secondary educational-level students, the lesser moral permissiviness; and the greater dissatisfaction with familiy functioning, the greater moral permissiveness.

\section{Introducción}

De la revisión de la literatura especializada se puede concluir que, el impresionante avance tecnológico ocurrido durante este siglo, ha producido numerosos cambios en el ámbito sociocultural, que han impactado fuertemente a las familias y a la sociedad y, que desafían la mantención de la cohesión social, de las tradiciones culturales y las pautas de comportamiento tradicionalmente aceptadas (Hoffman, París y Hall, 1997; Florenzano 1995; Alvarez, 1989). Entre los cambios que se han producido en los últimos años como producto de los procesos de modernización, diversos autores sostienen que se han afectado las orientaciones de valor de los individuos, los que se centran más en el logro individual que en los de tipo colectivo ( Merino, J.M., 1987). En el ámbito familiar, se observan cambios relacionados con el ejercicio de la autoridad; con la presencia o ausencia de los padres en el hogar (Valenzuela, 1995), y con una exposición de los jóvenes a riesgos crecientes de: embarazo adolescente, drogadicción, delincuencia, alcoholismo, fracaso y deserción escolar (Cooksey, 1997; Manlove, 1997; Fergusson, 1990, 1996; Wu, 1996; McLanahan, 1988, 1994). Podría hipotetizarse que esto se relaciona por un lado, con las orientaciones valóricas de los jóvenes y por otro, con los cambios en la estructura y funcionamiento que ha sufrido la familia en la sociedad actual. El cambio en los valores y desaparición de los modos tradicionales de hacer las cosas, ha sido reportado también en Chile en estudios realizados, entre otros, por Florenzano (1995).

En esta misma línea, al estudiar el comportamiento sexual de los estudiantes de la Universidad de Concepción, se detectó que las conductas de riesgo: iniciación temprana de las relaciones, alto número de parejas sexuales, relaciones sexuales con personas diferentes al "pololo" o novio y relaciones sin método de anticoncepción, se asociaban fuertemente con la moralidad de los estudiantes; que la moralidad, a su vez, se relacionaba con variables familiares y de filiación y práctica religiosa, y no con indicadores de status socioeconómico (Mathiesen et al., 1999). De estos hallazgos, surge el interés por estudiar uno de los aspectos de la moralidad: la permisividad personal, legal y sexual, en una población más heterogénea de jóvenes, como es la de los estudiantes de enseñanza media de la Provincia de Concepción, así como analizar cuál es el efecto de las variables de estructura y funcionamiento de la familia sobre la permisividad de estos estudiantes.

En este estudio se concibe la familia como la célula básica de la sociedad, que cumple, entre otras funciones vitales para su desarrollo y el de los individuos que forman parte de ella, con la función de ente principal transmisor de valores a través del proceso de endoculturación de sus nuevos miembros (Rice, 1997; Hoffman, Paris y Hall, 1997; Papalia, 1997; Craig, 1997). Siguiendo a Bronfenbrenner (1989) se la considera como un sistema abierto, que tiene una estructura y un funcionamiento y está integrada por personas con orientaciones de valor diversas, que juega un importante rol en la formación de los individuos. Su función educadora fundamental, responsable de transmitir las normas y valores sociales a sus descendientes y prepararlos para funcionar en el mundo social, ha sido reconocida en todas las culturas a través de la historia (Levy, 1975; Montenegro, 1995). La familia es el principal transmisor de los co- 
nocimientos, valores, actitudes, roles y hábitos que una generación pasa a la siguiente (Papalia, 1997). Es el agente de socialización, que junto con el colegio, los pares y los medios de comunicación social, contribuyen al logro de las tareas que el ser humano tiene que ir alcanzando en etapas anteriores a la adultez para llegar a ser integralmente desarrollado, capaz de autodirigirse, realizarse como persona y contribuir positivamente al desarrollo de los otros y de la sociedad en general ( Navarro, G., 2000). Ella permite al hombre cultivar todo lo que le hace "ser verdaderamente persona, haciéndose plenamente libre y responsable de sí mismo, de los otros significativos y del mundo en el que vive" (Elsner et al., 2000 pp.15).

Si se considera que la influencia familiar constituye parte de uno de los factores del desarrollo humano (factor ambiental) y siguiendo los planteamientos actuales de la psicología del desarrollo (Rice, 1997; Hoffman et al., 1997; Craig, 1997; Papalia, 1997) el entender a la familia como un subsistema social del que forman parte los jóvenes, quienes se han desarrollado como nuevos miembros de ella, permite visualizar como variables predictoras de las orientaciones de los jóvenes, algunas de estructura familiar y otras de funcionamiento o de proceso.

En cuanto a estructura, si bien existen diferentes posturas teóricas para el concepto de estructura familiar, para los fines de este estudio, se entiende como la composición del grupo familiar en el cual está inserto el estudiante, considerando variables como: tamaño, tipo, existencia de hermanos, posición ordinal, existencia de separaciones; además de otras características relativamente estables como nivel educacional y ocupacional de los padres, entre otras. Al respecto, según Elsner y colaboradores (2000) el aumento de separaciones matrimoniales que hoy se observa en Chile, podría atribuirse a un proceso de rápidos y profundos cambios en la sociedad, entre los que se cuenta la dis- minución del tiempo compartido al realizar gran parte de la vida fuera del hogar y un desequilibrio en los roles conyugales, configurado a partir de la incorporación de la mujer al mundo laboral.

Con relación al funcionamiento, en los últimos tiempos se ha ido produciendo un debilitamiento de las funciones tradicionales de la familia, como la menor importancia de la función educativa y de cuidado de los hijos, de la que es parte importante la formación de los valores, las que han sido, en gran parte, transferidas a otras instituciones sociales, como escuelas, jardines infantiles, salas cunas, entre otros, lo que ha contribuido significativamente a su pérdida de poder y a la generación de desajustes, desintegración de valores y conflicto de roles (Montenegro, 1995; Bernales, 1995).

Las familias tienen dificultad para adaptarse a los requerimientos de flexibilización de roles y modernización de su estructura $\mathrm{y}$ funcionamiento, especialmente frente a las nuevas formas de inserción social de las mujeres (Valenzuela, 1995) asunto que podría repercutir en la formación de los hijos, ahora tal vez con menor vigilancia de los padres y sometidos a mayores contactos con sus pares y con los medios de comunicación. Si bien hay estudios que indican que hijos de padres separados no presentan más problemas que hijos de parejas que se mantienen unidos, pero mal avenidas, diversos autores han observado que los hijos de padres separados presentan mayor frecuencia de alteraciones emocionales y conductuales que los hijos de parejas que permanecen unidas y bien avenidas, y que estos efectos son prolongados en el tiempo (Florenzano et al., 1995; Reyes y Muñoz, 1997, en Elsner et al., 2000). Por tanto, parece trascendente para la formación de las orientaciones de valor en los jóvenes, el funcionamiento de sus familias, independiente de cual sea su estructura, puesto que el funcionamiento familiar debería basarse en relaciones de solidaridad y re- 
ciprocidad, es decir, en familias en que éste es adecuado, los hijos además de tener sus necesidades satisfechas de manera más eficiente, estarían siendo socializados en el ejemplo de orientaciones hacia los otros, base de un adecuado funcionamiento de sus futuras familias de procreación, así como de una inserción social más satisfactoria. Almenar(1999) destaca la necesidad de estudiar científicamente los valores en los estudiantes de enseñanza media en España, en consideración a que éstos son el motor para la vida y los puntos de referencia que guían las actuaciones concretas de las personas y a que se adquieren con el ejercicio continuo; pensando además, que existe una preocupación por alcanzar maneras que hagan realidad, en las nuevas generaciones, una formación en los valores para la convivencia social armónica.

En este estudio, como variables de proceso, se considera el Funcionamiento Familiar, medido de manera global, a través del grado de insatisfacción del estudiante con las relaciones que se dan entre él y los miembros de su familia y con sus aspiraciones de protección y afecto $y$, a través de la percepción que declara el estudiante acerca del grado de interacción entre él y los otros integrantes del grupo familiar, y del afecto y ayuda recibida por él. También se evalúa el grado de compromiso y disposición a obedecer a los miembros de la familia, así como la afectuosidad y el autoritarismo del padre y de la madre o de los adultos a cargo, junto con algunas pautas de crianza, tales como: tipos de refuerzo, estilo educativo percibido y qué adulto otorga los permisos.

A pesar de que los estudiosos de la familia, han resaltado la importancia de los valores (Florenzano, 1995; Wu, 1996; McLanahan, 1994) los trabajos en esta línea se refieren a orientaciones en áreas específicas, como por ejemplo: personalidad y valores en adolescentes (Gómez, 1985), género y valores (Beutel y Mooney, 1995) valores y socialización infantil (Gerris et al.,
1997). No se detectó estudios empíricos que relacionen estructura y funcionamiento familiar con orientaciones valóricas de los adolescentes.

La moralidad, constituye un aspecto del ser humano que, como todas las áreas de su personalidad, se desarrolla gradualmente (Piaget, 1932 y Kohlberg, 1978, en Celedón, 1986). Está formada por diversos componentes: 1 - Habilidad de razonamiento general, la que constituye la capacidad de organizar y dar sentido a la información disponible en una situación de conflicto moral, es decir es un desarrollo estructural cognitivo (Piaget, 1967, en Kohlberg, 1987). 2 - Toma de perspectiva social, esto es la capacidad de darse cuenta que los otros tienen puntos de vista diferentes a los propios y comprender cómo estos se pueden relacionar y coordinar entre sí; es la habilidad de mirarse a sí mismo desde la perspectiva de los otros (Selman, 1980, en Vigneaux, 1990) es la que permite a la persona resolver en forma justa situaciones de conflicto moral, porque puede anticipar las necesidades de los demás y fomentar la justicia social y convivencia sana (Head, 1934, en Allende y col. 1989). 3- Juicio o razonamiento moral, para Kohlberg (en Vigneaux, 1990) es la habilidad para discernir entre lo que es correcto o incorrecto en una situación de dilema moral, es decir, en aquellas situaciones en que entran en conflicto valores apreciados por la persona. Este componente determina la acción por la vía de los derechos y deberes que surgen a partir de la situación. Al respecto, se ha observado que una mayor madurez de juicio moral aumenta la predisposición a comportarse moralmente (Mestre et al., 1999; Kohlberg, 1987; Colby et al., en Allende y col., 1989). 4- Factores afectivos, que son los encargados de energizar y motivar la conducta moral. Entre ellos los más estudiados han sido la empatía y la culpa, que son los componentes del desarrollo moral que preceden a la conducta de ayuda, actuando como elemen- 
tos motivadores de la conducta moral (Aderman y Berkowitz, 1970; Staub 1978; Hofman, 1980, citados en Allende et al., 1989). 5- Conducta moral, luego de una decisión en la que participan todos los factores anteriores, el hombre hace efectivo su desarrollo moral en una conducta moral, la que puede ser: conducta prosocial y altruista (actos voluntarios e intencionales para beneficiar a otros sin la anticipación de recompensas externas); conducta de cooperación (cuando dos o más personas trabajan para lograr una meta común, logrando una recompensa mayor que la que cada uno hubiera logrado por separado) y, autocontrol o inhibición de actos prohibidos (evitación de actos que hieran a otros, así como la capacidad para respetar normas sociales en ausencia de control externo) (Staub, 1978, en Vigneaux, 1990).

El propósito de este estudio es conocer, un aspecto de la moralidad: la Permisividad hacia determinados comportamientos, que se midieron a través de las apreciaciones del encuestado, sobre el grado en que considera aceptable ciertas conductas controvertidas legales, sexuales y de honorabilidad personal.

Además, se evalúa el efecto de variables de estructura y funcionamiento de las familias de los jóvenes estudiados, sobre su permisividad moral. A la vez que se inspecciona el efecto de la permisividad moral en algunos comportamientos y características psicosociales de los jóvenes.

\section{Metodología}

\section{Instrumentos}

1.Escala de Comportamientos Morales Controvertidos (The Morally Debatable Behaviors Scales) de Harding y Phillips, 1986 (en J. Robison et al, 1991) que mide el grado de permisividad respecto a tres factores: sexualidad, legalidad y honorabilidad personal. Fue utilizado en estudiantes universitarios (Mathiesen et al., 1998 y 1999) donde demostró su confiabilidad para ser aplicado en jóvenes y adultos jóvenes de Concepción; no obstante, dado que podría haber presentado problemas al aplicarlo en adolescentes, en este estudio también se analiza su funcionamiento. Se trata de un constructo de 22 itemes que expresan comportamientos moralmente cuestionables, acerca de los cuales el entrevistado debe manifestar su grado de aceptación, puntuando desde 1 que significa nunca aceptable, hasta 10, siempre aceptable. Esta escala puede ser usada como medida global. Además se divide en tres sub escalas que son las siguientes:

Moralidad sexual personal: con nueve itemes centrados en la vida, la muerte y las relaciones sexuales.

Moralidad personal $\mathrm{u}$ honorabilidad: con ocho itemes referidos a integridad frente a comportamientos de interés personal.

Moralidad legal: también con ocho reactivos, acerca de comportamientos proscritos por la ley.

2. Apgar Familiar de Smilkstein modificado, que evalúa el grado de interacción, apoyo y afecto que percibe el sujeto en su familia (Maddaleno, 1986).

3. Escala de funcionamiento familiar de Feetham (Roberts y Feetham, 1982) modificada por el equipo investigador, que mide insatisfacción con el funcionamiento familiar (Mathiesen, 1989, 1999).

4. Escala de familismo de Bardis (en De Miguel, 1967) mide compromiso, disposición a ayudar, a obedecer o estar cerca de los integrantes del sistema total parental, tanto del núcleo familiar como de la parentela. Puede ser 
usada con dos sub escalas, integración a la familia nuclear e integración de la familia extensa.

5. Escala de problemas juveniles percibidos, confeccionada por el equipo investigador, incluye 19 problemas sobre los que se pide al estudiante marcar cuáles afectan a la juventud actualmente, cuáles a alguno de sus amigos y por último al encuestado mismo.

6. Escala para medir grado de felicidad de Braudburn y Caplovits modificada por De Miguel (1967).

7. Encuesta, confeccionada ad hoc, para indagar acerca de la estructura familiar, la historia académica y algunas variables relacionadas con los roles paternales.

\section{Universo y Muestra}

Para este estudio el universo lo constituyeron los jóvenes que cursaban enseñanza media durante el año 1999 en los establecimientos de la provincia de Concepción.

Para seleccionar las unidades de análisis se utilizó el muestreo estratificado, al azar, estratificado por comunas y tipo de dependencia, en etapas, a partir del listado de instituciones de enseñanza media de la Secretaria Ministerial de Educación de la Octava Región.De cada uno de los establecimientos seleccionados, se tomó también al azar, un curso por nivel y dos alumnos por curso, uno de cada sexo. Se programó una muestra de 296 alumnos y se encuestó a la totalidad.

\section{Tabla 1}

Universo y Muestra de Establecimientos Educacionales de la Provincia de Concepción

\begin{tabular}{|c|c|c|c|c|c|c|c|c|}
\hline \multicolumn{9}{|c|}{ Tipo Establecimiento } \\
\hline \multirow[t]{2}{*}{ COMUNA } & \multicolumn{2}{|c|}{ Municipal } & \multicolumn{2}{|c|}{ Subvencionado } & \multicolumn{4}{|c|}{ No Subvencionado Adm.Delegada } \\
\hline & Total & Muestra & Total & Muestra & Total & Muestra & Total & Muestra \\
\hline Concepción & 8 & 4 & 7 & 3 & 12 & 6 & 4 & 2 \\
\hline Talcahuano & 7 & 3 & 2 & 1 & 4 & 2 & 1 & - \\
\hline Coronel & 3 & 1 & 1 & 1 & 1 & - & 1 & 1 \\
\hline Lota & 3 & 1 & 1 & 1 & - & - & - & - \\
\hline Penco & 1 & 1 & - & - & - & - & - & - \\
\hline Tomé & 2 & 1 & - & - & - & - & 1 & 1 \\
\hline Florida & 1 & 1 & - & - & - & - & - & - \\
\hline Hualquí & 1 & 1 & - & - & - & - & - & - \\
\hline Santa Juana & 1 & 1 & - & - & - & - & - & - \\
\hline Sn.Pedro de la Paz & 3 & 1 & 2 & 1 & 1 & - & - & - \\
\hline Chiguayante & 1 & 1 & 1 & 0 & 3 & 2 & - & - \\
\hline Total & 31 & 16 & 13 & 7 & 19 & 10 & 7 & 4 \\
\hline
\end{tabular}




\section{Procedimiento}

Se realizó una prueba piloto a 30 estudiantes pertenecientes a otros colegios de la zona no seleccionados, sin detectarse problemas para su aplicación. Se aseguró el anonimato y el secreto a los encuestados. La recolección de los datos se realizó durante los meses de Octubre y Noviembre del año 1999.

El análisis estadístico de los datos fue realizado por el equipo investigador utilizando el programa SAS (Statistical Analisis System).

\section{Resultados}

En primer lugar se presenta sintéticamente los resultados del funcionamiento de la escala de moralidad de Harding y Phillips, luego se describe la permisividad de los estudiantes de la muestra, en los tres aspectos que evalúa la escala en referencia. Por último se analiza las relaciones entre las variables seleccionadas para este estudio y la permisividad moral.

\section{1-Características Psicométricas del Instrumento}

1.1 La confiabilidad global del instrumento con el coeficiente alfa de Cronbach, resultó adecuada: 0.73 para la escala total, levemente inferior al 0.79 detectado en universitarios. Las tres sub escalas también fueron confiables: 0.70 para permisividad legal y 0.72 para permisividad personal y sexual.

La consistencia interna de la escala, medida por la vía de correlacionar cada ítem con el total de la escala, fue adecuada, todos se correlacionaron positiva y significativamente con la escala total (p todas inferiores a 0.0001 y r entre 0.23 y 0.63 , la mayoría sobre $0.50)$. La consistencia interna de las sub-escalas resultó alta; todos los ítemes tuvieron correlación positiva y altamente significativa con los sub totales de las escalas respectivas ( todas con $p=0.0001$ ). En la de permisividad personal las correlaciones $\mathrm{r}$ de Pearson fluctuaron entre 0.37 y 0.60 , la gran mayoría estuvo sobre 0.50 . En la de permisividad sexual ocurre casi lo mismo, las $\mathrm{r}$ se ubicaron entre 0.45 y 0.62 . Con respecto a la sub-escala legal, todas las correlaciones ítem sub escala fueron superiores a 0.47.

Las correlaciones de las sub-escalas con la escala total, fueron de 0.83 , para la de permisividad moral personal, 0.85 para la sub-escala legal y 0.86 para permisividad sexual. Estos resultados son también indicativos de la consistencia de este instrumento.

1.2 En este estudio no se evaluó la validez de esta escala puesto que fue inspeccionada en el estudio en alumnos universitarios al que ya se hizo referencia. Allí se demostró validez convergente y coherencia teórica, ambas indicativas de adecuada validez (Mathiesen et al., 1998).

1.3 Por último, el rango de variabilidad detectado fue el máximo en todos los itemes, lo que muestra adecuada capacidad discriminatoria.

\section{2-La permisividad moral del estudiante de enseñanza media de la provincia de Concepción.}

Se detectó un promedio total de permisividad para los alumnos estudiados, de 2.97, con un mínimo de 1.05 y un máximo de 9.14 (como ya se señaló, el 1 es nunca permitido y el 10 siempre permitido). Este promedio total es levemente inferior al 3.27 reportado para los estudiantes universitarios (op. cit.). Los promedios por sub-escalas fueron: el más bajo, en permisividad personal con 2.73 , con un mínimo de 1.2 y 
un máximo de 8.7; mientras que el más alto se detectó en permisividad sexual, con 3.16 y un rango de variación entre 1.5 y 10; el promedio de moralidad legal se ubica en posición intermedia con 2.79 y 1.3 de puntaje mínimo y 8.7 de máximo. En los estudiantes universitarios, la menor permisividad fue legal, y la mayor, al igual que en estos estudiantes, se da en el ámbito de los comportamientos sexuales.

En la tabla 2 se aprecia que los itemes cuyo promedio de permisividad es más bajo, en orden creciente, son: el aborto, el fumar marihuana, sacar auto ajeno sin per- miso. La mayor permisividad se ubica en matar en defensa propia, seguida de guardar dinero encontrado, no cancelar tarifa en el transporte público, el divorcio y las relaciones sexuales en menores de edad.

El promedio de aceptación de la eutanasia es más alto que el de comportamientos similares, referidos a suprimir vidas, como el suicidio y el aborto y, evadir impuestos o infidelidad, que han sido tradicionalmente de mayor aceptación social y que implican frente a la ley chilena delitos menos graves que el asesinato, por muy caritativo que éste pretenda ser.

Tabla 2

Promedios, desviaciones de permisividad por conducta cuestionable, ordenados de mayor a menor permisividad.

\begin{tabular}{lll}
\hline & X & s \\
\hline 21. Matar en defensa propia & 5.60 & 3.49 \\
7. Conservar dinero encontrado & 4.48 & 3.03 \\
2. No cancelar tarifa en el transporte público & 4.38 & 3.18 \\
15. Divorcio & 4.28 & 2.91 \\
10 Tener relaciones sexuales antes de la mayoría de edad & 4.20 & 3.09 \\
17. Eutanasia & 3.06 & \\
16. Pelear con la policía & 3.43 & 2.70 \\
19. No informar daño causado a vehículo estacionado & 3.19 & 2.37 \\
8. Mentir por su propio interés & 3.07 & 2.55 \\
1. Reclamar beneficios estatales que no corresponden & 2.95 & 2.56 \\
3. Evadir impuesto si hay oportunidad de hacerlo & 2.85 & 2.64 \\
12. Homosexualidad & 2.47 & 2.44 \\
18. Suicidio & 2.23 & 2.38 \\
22. Asesinatos políticos & 2.22 & 2.30 \\
9. Hombres o mujeres casados teniendo aventuras & 2.20 & 2.28 \\
20. Amenazar trabajadores rehusan participar huelga & 2.19 & 2.20 \\
4. Comprar algo sabiendo que es robado & 2.11 & 2.03 \\
11. Aceptar soborno en el curso de sus obligaciones & 2.11 & 2.11 \\
13. Prostitución & 2.09 & 2.13 \\
5. Tomar vehículo sin permiso, (paseo alocado). & 1.98 & 1.86 \\
6. Fumar marihuana o hashish. & 1.88 & 1.94 \\
14. Aborto & 1.78 & 1.97 \\
Total & 2.97 & 1.19 \\
\hline
\end{tabular}




\section{3-Relaciones de la permisividad moral con otras variables.}

Siguiendo a Williams (1979) primero se analiza la permisividad moral como variable dependiente y luego, se presenta los efectos de la moralidad sobre algunas dimensiones de los jóvenes.

\section{1- Permisividad moral según variables} que caracterizan al joven.

En la tabla tres, se aprecian diferencias significativas en la moralidad total según el sexo que demuestran una mayor permisividad en los hombres. Por subescalas, las di- ferencias entre hombres y mujeres se mantienen en moralidad legal y aumentan en la moralidad personal, mientras que desaparecen en permisividad sexual; diferencias en el mismo sentido se detectaron en los universitarios (op cit). Por otra parte, Tamayo (1993) también informa de diferencias valóricas en adolescentes según sexo. Con la edad hubo sólo una leve tendencia a la correlación inversa: a mayor edad menor permisividad, pero sin significación estadística, probablemente debido al bajo rango de variabilidad de la edad. Tampoco hubo diferencias significativas según el curso al que asistía el estudiante, probablemente por si-

Tabla 3

Permisividad Moral Global y por Dimensiones según Sexo y Religión del Estudiante de Enseñanza Media de la Provincia de Concepción

\begin{tabular}{|c|c|c|c|c|}
\hline Variable & Promedio & $\mathbf{n}$ & Prueba & $p$ \\
\hline Sexo & Global & & \multirow{3}{*}{$t=1.96$} & \multirow{3}{*}{.05} \\
\hline Mujeres & 2.83 & 148 & & \\
\hline Hombres & 3.10 & 148 & & \\
\hline $\begin{array}{l}\text { Mujeres } \\
\text { Hombres }\end{array}$ & $\begin{array}{l}\text { Personal } \\
2.56 \\
2.91\end{array}$ & 148 & $\mathrm{t}=2.42$ & .02 \\
\hline $\begin{array}{l}\text { Mujeres } \\
\text { Hombres }\end{array}$ & $\begin{array}{l}\text { Legal } \\
2.64 \\
2.95\end{array}$ & $\begin{array}{l}148 \\
148\end{array}$ & $\mathrm{t}=2.00$ & .05 \\
\hline $\begin{array}{l}\text { Mujeres } \\
\text { Hombres }\end{array}$ & $\begin{array}{l}\text { Sexual } \\
3.06 \\
3.25\end{array}$ & $\begin{array}{l}148 \\
148\end{array}$ & $\mathrm{t}=1.03$ & .3 N.S. \\
\hline Religión & Global & & \multirow{4}{*}{$\mathrm{F}=2.62$} & \multirow{4}{*}{.07} \\
\hline Ninguna & $3.34 \mathrm{~A}$ & 35 & & \\
\hline Católicos & $2.98 \mathrm{AB}$ & 168 & & \\
\hline Otras & $2.81 \mathrm{~B}$ & 93 & & \\
\hline $\begin{array}{l}\text { Ninguna } \\
\text { Católica } \\
\text { Otras }\end{array}$ & $\begin{array}{l}\text { Personal } \\
2.97 \\
2.71 \\
2.70\end{array}$ & $\begin{array}{r}35 \\
168 \\
93\end{array}$ & $\mathrm{~F}=0.73$ & .49 N.S \\
\hline $\begin{array}{l}\text { Ninguna } \\
\text { Católicos } \\
\text { Otras }\end{array}$ & $\begin{array}{l}\text { Legal } \\
2.82 \\
2.81 \\
2.73\end{array}$ & $\begin{array}{r}35 \\
168 \\
93\end{array}$ & $\mathrm{~F}=.11$ & .90 N.S \\
\hline & Sexual & & \multirow{4}{*}{$F=6.61$} & \multirow{4}{*}{.002} \\
\hline Ninguna & $3.91 \mathrm{~A}$ & 35 & & \\
\hline Católicos & $3.18 \mathrm{~B}$ & 168 & & \\
\hline Otras & $2.83 \mathrm{~B}$ & 93 & & \\
\hline
\end{tabular}


milar. En cuanto a la religión del estudiante, si se acepta un $7 \%$ de probabilidad de error, se puede observar que los jóvenes que se declararon evangélicos o de otras religiones tuvieron un promedio significativamente más bajo de permisividad que aquellos sin religión; el promedio de los católicos se ubicó en posición intermedia. Respecto a la moralidad sexual, la significación aumenta $\mathrm{y}$ se pierde en moralidad legal y personal. Estos resultados concuerdan con lo informado para estudiantes universitarios, donde los que declaraban no tener credo religioso eran los que tenían proporciones significativamente mayores de conductas sexuales de riesgo, (Mathiesen et al., 1999).

\subsection{Permisividad Moral según Estruc- tura Familiar.}

No se detectaron diferencias según variables de estructura familiar: tipo y tamaño de la familia, estado civil de los padres, número de hermanos, número de años de convivencia con la pareja actual de los padres. Sólo se observó una tendencia a mayor permisividad sexual en los hijos de solteras.

El estatus socio económico familiar, al medirlo por el número de bienes de la familia, se relacionó con la moralidad del estudian- te; cuando era alto, el promedio de permisividad era mayor: a mayor número de bienes que posee la familia, más permisividad moral. Al especificar por sub-escalas, la correlación con moralidad legal y personal desaparece, y aumenta para la permisividad sexual. En cuanto al tipo de colegio al que asistían los jóvenes, hubo diferencias significativas: los alumnos de colegios particulares no subvencionados presentaron un promedio de permisividad más alto que todos los otros grupos; en las subescalas, se mantiene este mismo resultado sólo en permisividad sexual. La educación de la madre no presentó relaciones significativas con la moralidad, sólo una tendencia a mayor permisividad sexual en los hijos de las madres de educación alta. En el caso de la educación del padre se dio la misma tendencia para la permisividad global y por sub-escalas, alcanzando significación en permisividad sexual, siendo el promedio más alto de permisividad sexual el de los hijos de padres con educación alta, y el más bajo, el de aquellos cuyos padres tenían educación baja; todas las otras categorías están en posición intermedia sin diferencias significativas entre sí $(\mathrm{F}=2.37 \mathrm{p}=.04)$. Esta tendencia a mayor permisividad sexual en los estudiantes pertenecientes al estrato más alto, no se encontró en los universitarios. 


\section{Tabla 4}

Permisividad Moral del Estudiante de Enseñanza Media según Indicadores de Nivel Socio-Económico. $(n=296)$

\begin{tabular}{|c|c|c|c|}
\hline Variable Independiente & Variable Dependiente & Prueba & $\mathbf{p}$ \\
\hline $\begin{array}{l}\mathrm{N}^{\circ} \text { de bienes } \\
\text { Tipo de Colegio }\end{array}$ & $\begin{array}{l}\text { Moral Global } \\
\text { Moral Personal } \\
\text { Moral Legal } \\
\text { Moral Sexual } \\
\text { Promedio/Tipo }\end{array}$ & $\begin{array}{l}r=.16 \\
r=.05 \\
r=.07 \\
r=.24\end{array}$ & $\begin{array}{l}.005 \\
.41 \mathrm{~N} . \mathrm{S} \\
.21 \mathrm{~N} . \mathrm{S} \\
.0001\end{array}$ \\
\hline $\begin{array}{l}\text { Part. No Subvencionado } \\
\text { Part. Subvencionado } \\
\text { Adm. Delegada } \\
\text { Municipal }\end{array}$ & $\begin{array}{lr}\text { Moral Global } & \mathbf{n} \\
3.33 \mathrm{~A} & 80 \\
2.86 \mathrm{~B} & 56 \\
2.86 \mathrm{~B} & 32 \\
2.81 \mathrm{~B} & 128\end{array}$ & $\mathrm{~F}=3.63$ & .01 \\
\hline $\begin{array}{l}\text { Part. No Subvencionado } \\
\text { Adm. Delegada } \\
\text { Part. Subvencionado } \\
\text { Municipal }\end{array}$ & $\begin{array}{l}\text { Moral Personal } \\
2.97 \\
2.71 \\
2.66 \\
2.62\end{array}$ & $\mathrm{~F}=1.31$ & .27 N.S \\
\hline $\begin{array}{l}\text { Part. No Subvencionado } \\
\text { Adm. Delegada } \\
\text { Part. Subvencionado } \\
\text { Municipal }\end{array}$ & $\begin{array}{l}\text { Moral Legal } \\
3.03 \\
2.86 \\
2.73 \\
2.66\end{array}$ & $\mathrm{~F}=1.31$ & .27 N.S. \\
\hline $\begin{array}{l}\text { Part. No Subvencionado } \\
\text { Part. Subvencionado } \\
\text { Municipal } \\
\text { Adm. Delegada }\end{array}$ & $\begin{array}{l}\text { Moral Sexual } \\
3.74 \mathrm{~A} \\
3.03 \mathrm{~B} \\
2.92 \mathrm{~B} \\
2.85 \mathrm{~B}\end{array}$ & $F=5.80$ & .0007 \\
\hline $\begin{array}{l}\text { Educación del Padre } \\
\text { Universitaria } \\
\text { Básica Incompleta } \\
\text { Media Completa } \\
\text { Básica Completa } \\
\text { Media Incompleta }\end{array}$ & $\begin{array}{l}\text { Moral Sexual } \\
3.62 \mathrm{~A} \\
3.14 \mathrm{BA} \\
3.10 \mathrm{BA} \\
2.80 \mathrm{~B} \\
2.80 \mathrm{~B}\end{array}$ & $\mathrm{~F}=2.60$ & .04 \\
\hline
\end{tabular}

\subsection{Permisividad moral según Funcio- namiento Familiar.}

Se encontró asociación de todos los indicadores de funcionamiento familiar con la permisividad moral; se detectó una co- rrelación positiva entre la insatisfacción con el funcionamiento familiar y la aceptación de comportamientos cuestionables: a mayor insatisfacción con el funcionamiento familiar mayor permisividad moral global y por 
subescalas. Esta relación de la permisividad con la insatisfacción aumenta con la subescala específica de insatisfacción con las relaciones familiares; mientras que desaparece en las otras sub-escalas, excepto en la de insatisfacción con el tiempo para dedicar a los deberes escolares y al ocio en que se detectó correlación significativa con la permisividad sexual: a mayor insatisfacción con el tiempo disponible, mayor permisividad sexual.

Se encontró una correlación inversa entre el grado de interacción y apoyo familiar (Apgar familiar) con la permisividad: a mayor interacción y apoyo familiar menor acep- tación de conductas cuestionables. Para moralidad total y moralidad sexual, se encontraron correlaciones bajas pero altamente significativas. Con la moralidad personal desaparece la relación y con la legal disminuye.

La misma tendencia se da entre Familismo y permisividad: a mayor familismo menor permisividad. Esta correlación no alcanza significación en la moralidad total $(p=.06)$, no existe en moralidad legal y personal, mientras que aumenta su fuerza y adquiere significación en moralidad sexual.

Tabla 5

Correlaciones (r de Pearson) de Variables de Funcionamiento Familiar con Permisividad Moral. $(n=296)$.

\begin{tabular}{llll}
\hline Variable Independiente & Variable Dependiente & $\mathbf{r}$ & $\mathbf{p}$ \\
\hline Insatisfacción Total & Moral Global & .17 & .003 \\
& Moral Personal & .12 & .04 \\
& Moral Legal & .14 & .02 \\
& Moral Sexual & .14 & .02 \\
Insatisfacción Familiar & Moral Global & .20 & .0004 \\
& Moral Personal & .15 & .01 \\
& Moral Legal & .15 & .02 \\
& Moral Sexual & .18 & .001 \\
Insatisfacción Tiempo & Moral Sexual & .12 & .03 \\
Interacción Familiar & Moral Global & -.15 & .009 \\
& Moral Personal & -.09 & $.12 \mathrm{~N} . S$. \\
& Moral Legal & -.11 & $.07 \mathrm{~N} . \mathrm{S}$. \\
Familismo Global & Moral Sexual & -.16 & .007 \\
& Moral Global & -.11 & $.06 \mathrm{~N} . \mathrm{S}:$ \\
& Moral Personal & -.02 & $.76 \mathrm{~N} . \mathrm{S}$. \\
& Moral Legal & -.03 & .64 N.S. \\
& Moral Sexual & -.18 & .002 \\
Familiasmo hacia & Moral Global & -.15 & .01 \\
Familia Nuclear & Moral Personal & -.04 & $.51 \mathrm{~N} . \mathrm{S}$. \\
& Moral Legal & -.04 & .48 N.S. \\
& Moral Sexual & -.23 & .0001 \\
\hline
\end{tabular}


Otros de los aspectos de funcionamiento familiar considerados en el estudio son algunos relacionados con la crianza, como: tipos de refuerzo, grado de autoritarismo y de afectuosidad de los padres y estilo educativo. En cuanto al tipo de castigo, cerca de la mitad de los estudiantes declaran que no los castigan nunca, de los que respondieron que si los castigaban, el tipo de castigo se relacionó con la permisividad global, así como con las tres específicas; los que son enviados a la pieza o a la cama son los que presentaron el promedio significativamente más alto; todas las otras categorías de castigo (reto, sin salida, sin mesada, quitan otros privilegios, les hablan y los golpean) no son significativamente diferentes entre sí. Por otra parte, no hubo diferencias significativas según el tipo de premio con que se refuerzan los buenos comportamientos, sólo una tendencia a mayor permisividad en los alumnos que recibían premios materiales. En cuanto a la afectuosidad de los padres, aún cuando las diferencias no alcanzaron significación, la tendencia fue muy clara, tanto en permisividad global como sexual, correspondió la menor permisividad a los hijos de madres cariñosas o muy cariñosas y la mayor a los de madres poco o nada cariñosas ( $p=.06$ y $p=.08$ respectivamente). No se detectaron diferencias en la permisividad según el autoritarismo de los padres percibido por los hijos (desde muy autoritarios hasta nada autoritarios). Con respecto al estilo educativo del hogar, se pidió al estudiante que eligiera de una lista cuál era el de su casa: tampoco hubo diferencias significativas, sin embargo, en el caso de la permisividad personal, si se aceptó una p de .07, los hijos de padres que actuaban "según el humor del momento", presentaron un promedio de permisividad mayor que los de padres estrictos; los otros estilos educativos tuvieron promedios intermedios. Con la permisividad global la tendencia es la misma pero pierde significación. 
Tabla 6

Promedios de Permisividad Moral (con diferencias significativas) según algunas pautas de Crianza.

\begin{tabular}{|c|c|c|c|c|}
\hline \multirow{2}{*}{$\begin{array}{l}\text { Variable Independiente } \\
\text { Tipo de Castigo }\end{array}$} & \multicolumn{2}{|c|}{ Variable Dependiente } & \multirow[t]{2}{*}{ Prueba F } & \multirow[t]{2}{*}{$\mathbf{p}$} \\
\hline & Moral Global & $\mathrm{n}$ & & \\
\hline & $5.49 \mathrm{~A}$ & 4 & 4.28 & .0009 \\
\hline Ninguno & $3.14 \mathrm{~B}$ & 46 & & \\
\hline Le Hablan & 2.92 B19 & & & \\
\hline No Responde & $2.91 \mathrm{~B}$ & 138 & & \\
\hline Quitan Privilegios & $2.87 \mathrm{~B}$ & 60 & & \\
\hline \multirow[t]{2}{*}{ Reto } & $2.84 \mathrm{~B}$ & 27 & & \\
\hline & \multicolumn{2}{|l|}{ Moral Personal } & & \\
\hline La Pieza & $4.94 \mathrm{~A}$ & 4 & 3.28 & .007 \\
\hline Ninguno & $2.97 \mathrm{~B}$ & 46 & & \\
\hline Reto & $2.71 \mathrm{~B}$ & 27 & & \\
\hline No Responde & $2.71 \mathrm{~B}$ & 138 & & \\
\hline Quitan Privilegios & $2.56 \mathrm{~B}$ & 60 & & \\
\hline \multirow[t]{2}{*}{ Le Hablan } & $2.51 \mathrm{~B}$ & 19 & & \\
\hline & \multicolumn{2}{|l|}{ Moral Legal } & & \\
\hline La Pieza & $5.34 \mathrm{~A}$ & 4 & 3.56 & .004 \\
\hline Ninguno & $2.94 \mathrm{~B}$ & 46 & & \\
\hline No Responde & $2.80 \mathrm{~B}$ & 138 & & \\
\hline Le Hablan & $2.68 \mathrm{~B}$ & 19 & & \\
\hline Quitan Privilegios & $2.61 \mathrm{~B}$ & 60 & & \\
\hline \multirow[t]{2}{*}{ Reto } & $2.60 \mathrm{~B}$ & 27 & & \\
\hline & \multicolumn{2}{|l|}{ Moral Sexual } & & \\
\hline La Pieza & $5.97 \mathrm{~A}$ & 4 & 3.27 & .007 \\
\hline Ninguno & $3.32 \mathrm{~B}$ & 46 & & \\
\hline Le Hablan & $3.26 \mathrm{~B}$ & 19 & & \\
\hline Quitan Privilegios & $3.15 \mathrm{~B}$ & 60 & & \\
\hline No Responden & $3.03 \mathrm{~B}$ & 138 & & \\
\hline Reto & $2.96 \mathrm{~B}$ & 27 & & \\
\hline Estilo Educativo & \multicolumn{2}{|l|}{ Moral Personal } & & \\
\hline Según Humor & $3.28 \mathrm{~A}$ & 25 & 2.32 & .06 \\
\hline Permisivo & $3.01 \mathrm{AB}$ & 14 & & \\
\hline Intermedio & $2.76 \mathrm{AB}$ & 152 & & \\
\hline Democrático & $2.60 \mathrm{AB}$ & 70 & & \\
\hline Estricto & $2.32 \mathrm{~B}$ & 27 & & \\
\hline
\end{tabular}


3.4 Posibles efectos de la permisividad moral del estudiante sobre algunas de sus características o comportamientos.

Al relacionar algunas dimensiones psicosociales del joven tales como: percepción de su calidad como estudiante, percepción de su popularidad y grado de felicidad, no se encontró ninguna asociación significativa, por tanto al parecer la permisividad moral no afecta estas dimensiones.

También se analizó el efecto de la permisividad moral sobre el rendimiento académico: promedio de notas del año anterior y del semestre anterior, número de notas altas, número de notas bajas, existencia de repitencias y cantidad de repitencias; no se detectó ninguna relación entre la permisividad moral y los resultados académicos.

En cambio, si se encontró relación directa de la permisividad moral del estudiante, con la cantidad de problemas que percibe como propios de sus amigos o la cantidad de problemas que lo afectan a él mismo, es decir, a mayor permisividad, mayor cantidad de problemas personales y de los amigos. Por otra parte, no se encontró correlación de la permisividad del estudiante con la cantidad de problemas percibidos por él como característicos de la juventud actual.

Tabla 7

Correlaciones ( $\mathrm{r}$ de Pearson) de la Moralidad del estudiante con la Cantidad de Problemas que percibe como propios y de sus amigos. $(\mathrm{n}=296)$

\begin{tabular}{llll}
\hline Variable Independiente & Variable Dependiente & r & p \\
\hline Moral Global & Problemas Amigos &. $\mathbf{1 8}$ & .002 \\
Moral Personal & & .12 & .04 \\
Moral Legal & & .12 & .04 \\
Moral Sexual & & 19 & .0009 \\
& Problemas Propios & .23 & \\
Moral Global & & .17 & .0001 \\
Moral Personal & & .20 & .003 \\
Moral Legal & & .21 & .0003 \\
Moral Sexual & & & \\
\hline
\end{tabular}

\section{Discusión y Conclusiones}

En primer lugar, se puede concluir que, tanto la escala de permisividad total como sus tres subescalas, son confiables. Esto se demostró con: las alpha de Crombach, las correlaciones itemes/escala total, itemes/ subescala, subescalas/escala total y subescalas entre si, todas ellas positivas y altamente significativas, lo que permite afir- mar que esta escala de permisividad moral puede ser utilizada en adolescentes de la provincia o de otras con similares características.

En segundo lugar, en cuanto a la moralidad detectada en los estudiantes de enseñanza media de la provincia de Concepción, se puede afirmar que se declaran como menos permisivos en moralidad personal y 
más permisivos en moralidad sexual. Sus niveles de permisividad son en general bajos, la mayoría de los comportamientos analizados están bajo los tres puntos promedio de aceptación. Especialmente baja aceptación presentaron: el aborto, la drogadicción, el sacar auto ajeno sin permiso; la mayor aceptación, en orden decreciente, fue para: matar en defensa propia, conservar dinero encontrado, no cancelar el transporte público, el divorcio y tener relaciones antes de la mayoría de edad.

Entre las variables del estudiante analizadas, sólo el sexo del joven explica su permisividad moral; los hombres presentaron un promedio de permisividad total y por niveles más alto. Aún cuando la tendencia fue la misma, no se detectaron diferencias significativas en moralidad sexual. A este respecto en el estudio anterior, ya citado, los estudiantes universitarios estudiados en 1995 (Mathiesen, et al., 1998) presentaron mayor permisividad sexual que sus compañeras del género femenino. Esto estaría indicando cambios en el sentido de una creciente permisividad sexual de las mujeres.

Ninguna de las variables tradicionalmente consideradas como de estructura, se asoció significativamente con la permisividad moral, lo que estaría indicando que, al menos en relación al efecto sobre la variable estudiada, no habría un tipo de familia mejor o peor que otro. De las otras dimensiones que en este estudio se consideraron como estructurales, el estatus socio económico de la familia, representado por la cantidad de bienes del hogar y el tipo de colegio al que asiste el estudiante, fue la única que mostró alguna relación con la moralidad, en el sentido que se asoció el mayor estatus económico con mayor permisividad moral, especialmente en el ámbito sexual. Esto podría estar asociado a una tendencia a mayor permisividad sexual en los hijos de las madres y padres de educación alta, quienes son los que tienen mayores bienes en el hogar e hijos en colegios particulares y probablemente son quienes tienden a crear hogares con culturas modernas, caracterizadas por la participación, relaciones democráticas, igualdad de género, mayor tolerancia, mayor aceptación de la diversidad, lo que podría estar influyendo en una mayor permisividad de los hijos, especialmente en el ámbito sexual, en las hijas.

Lo anterior no se encontró en los estudiantes universitarios estudiados el año 1995, lo que podría explicarse por una mayor diversidad en las culturas familiares de los estudiantes de enseñanza media y por tanto, sería necesario en futuros estudios, profundizar con mediciones más detalladas del nivel socio económico, su relación con la cultura familiar y de ésta con la permisividad.

El trabajo fuera del hogar de la madre no influye directamente en la moralidad, pues los hijos de las dueñas de casa presentaron un promedio de permisividad intermedio, no significativamente diferente del de los hijos de madres con otras ocupaciones, antecedente importante a considerar a la hora de hacer difusión masiva de creencias que no tienen fundamento empírico, pues ello podría ser un factor generador de estrés en las madres que trabajan fuera del hogar (porcentaje alto en la actualidad) $y$, por consiguiente un factor que interfiera en el funcionamiento familiar, variable que sí se correlacionó significativamente con la moralidad del estudiante en esta investigación.

Se encontró correlación directa entre moralidad e insatisfacción con el funcionamiento familiar: a mayor insatisfacción mayor permisividad; correlación inversa con el grado de interacción familiar: a mayor interacción menor permisividad; una tendencia en este mismo sentido inverso entre permisividad y familismo, significativa sólo con la permisividad sexual y más fuerte con el familismo hacia la familia nu- 
clear. Esto permite concluir que los jóvenes satisfechos con el funcionamiento de sus familias, independientemente de la estructura que ésta tenga, pertenecientes a familias cuyo grado de interacción es alto y que sienten apego y compromiso hacia sus parientes, presentan menor permisividad moral.

En otro ámbito del funcionamiento, el de las normas de crianza, hubo diferencias de la permisividad moral según el estilo educativo, aquel en que los padres sancionan según su humor, presentó el promedio significativamente más alto de permisividad personal, mientras que el promedio de los hijos de padres estrictos fue el más bajo; los estilos: permisivo, intermedio y democrático, ocuparon ese mismo orden pero sin diferencias significativas del primero ni del último. Pareciera ser que la percepción que tienen los jóvenes acerca del estilo de crianza de sus padres, en términos de normas claras y consistentes (estrictos), influye en la permisividad y que sus creencias frente a los otros estilos consultados probablemente excluyen la idea de normas claras y consistentes. Sería recomendable, en futuros estudios, aclarar a los jóvenes qué se está entendiendo por cada estilo o bien, no pedir que lo clasifiquen sino que lo describan.

Es importante destacar que cerca de la mitad de los estudiantes declaran que no los castigan nunca, lo que estaría indicando un cambio positivo en las creencias acerca de la educación de los hijos, en comparación con generaciones anteriores. Sin embargo, en este estudio no queda claro, por una dificultad del instrumento aplicado, si este cambio es a favor de un estilo más apropiado para el desarrollo psicológico, cual es el "autoritativo" o es a favor de estilos permisivos o negligentes.

El tipo de castigo se relacionó con la permisividad global, así como con las tres específicas; los jóvenes que son enviados a la pieza o a la cama son los que presentaron el promedio significativamente más alto. Todas las otras categorías de castigo (reto, sin salida, sin mesada, quitan otros privilegios, les hablan y los golpean) no son significativamente diferentes entre sí. Probablemente esto se relacione con el hecho que, por lo común, esta conducta de los padres no va asociada a una actitud educativa, a un análisis y explicación de la conducta inadecuada ni de los beneficios y consecuencias negativas de diferentes opciones o alternativas. Sin embargo, estos resultados deben ser tomados con precaución y merecen futuros estudios antes de llegar a conclusiones seguras, puesto que los estudiantes pertenecientes a esa categoría eran muy pocos, además, hubo más de un tercio de los entrevistados que no respondió esta pregunta, por tanto, en futuros estudios debería modificarse la forma de medición de esta variable. El tipo de premio no se asoció con la permisividad.

En cuanto a la afectuosidad de los padres, aún cuando las diferencias no alcanzaron significación, hay tendencia a menor permisividad en los hijos de madres cariñosas o muy cariñosas y la mayor en los de madres poco o nada cariñosas.

No se detectó ninguna relación entre la permisividad moral y los resultados académicos, lo que estaría indicando claramente que el ser más o menos permisivo en lo moral, no tiene relación con el éxito ni con los avances en el ámbito escolar.

Se encontró relación directa de la permisividad moral del estudiante, con la cantidad de problemas que percibe como propios de sus amigos o la cantidad de problemas que lo afectan a él mismo, es decir, a mayor permisividad, mayor cantidad de problemas personales y de los amigos. Por otra parte, no se encontró correlación de la permisividad del estudiante con la cantidad de problemas percibidos por él como característicos de la juventud actual. Parecie- 
ra que la permisividad moral ofrece al joven más alternativas de opción frente a eventos cotidianos, enfrentándolo a decisiones entre alternativas igualmente válidas para él, lo que lo llevaría a autopercibirse con más problemas vitales, lo que podría influir, por profecía autocumplida, en una mayor cantidad de conductas de riesgo, relación que sería necesario investigar.

Los resultados de esta investigación corroboran la importancia de la dinámica y funcionamiento familiar en el desarrollo de la moralidad, específicamente en lo relativo a la permisividad de los jóvenes y en la percepción de problemas en su vida. Si se considera que en estudios anteriores se comprobó que las conductas sexuales de riesgo se asocian principalmente con las orientaciones de valor (Mathiesen et al., 1999), entonces, el apoyo a la familia, por parte de diferentes instituciones sociales, para una mejor convivencia y dinámicas de funcionamiento, pasa a constituirse en una buena alternativa como factor protector de estas conductas de riesgo.

\section{Referencias Bibliográficas}

Almenar, N. (1999) Los Valores en los Jóvenes: Estrategias de Diagnóstico PAIDEA., 26: 9-30. Concepción, Chile.

Allende M. et al (1989) Desarrollo Moral y Familiar: Una Visión Integrativa y Estrategias de Estimulación. Tesis para optar al título de Psicólogo, Pontificia Universidad Católica de Chile.

Alvarez , M. (1989) Cambio Social y Familia. Revista de Sociología, Universidad de Chile, 4: 23-30.

Beutel, A. M. y, Mooney M.M. (1995) Gender and values. American Sociological Review, 60: 436-448
Bronfenbrenner, U. (1989) Ecological Systems Theory. Annals of Child Development, 6: 187-249.

Bernales M. S. (1995) Las Relaciones Familiares en el Chile de los 90. Proposiciones, 26: 13-33.

Celedón, M. (1986) Diseño y Aplicación de un Programa Educativo para desarrollar el Razonamiento Moral y las Estrategias de Enfrentamiento a Problemas Morales, en adolescentes chilenos de clase media. Tesis para optar al Título de Psicólogo. Santiago, Pontificia Universidad Católica de Chile.

Coleman, M., Ganong, L. y Cable, S. (1997) Beliefs about women's intergenerational family obligations to provide support before and after divorce and remarriage. Journal of marriage and family, 59: 165-176.

COOKSEY ,E. (1997) Consequences of Young mothers'marital histories for children'cognitive developement. Journal of Marriage and the Family, 59: 245261.

Craig, G. (1997) Desarrollo Psicológico. Ciudad de México, México. Prentice Hall.

De Miguel , A. (1967) Tres estudios para un Sistema de Indicadores Sociales. Madrid, España. Fundación FOESSA Euramérica.

Elsner, P. , Montero, M. de la L., Reyes, C. y ZEgers, B. (2000) La Familia una Aventura. Santiago, Chile. Pontificia Universidad Católica de Chile.

Fergusson, D.M. ,HorWood, L. y LaWTON, J. (1990) Vulnerability to childhood problems and family social bakground. Journal of Child Psychology and Psychiatry, 31, 7: 1145-1160.

Fergusson, D. y Lynskey, M. (1996) Adolescent Resiliency to Family Adversity. Journal of Child Psychology and Psychiatry, 37: $281-291$. 
Valores Morales y Familia..., Vol. XI, № 2: Pág. 55-74. 2002

Florenzano, R. (1994) Familia y Salud de los Jóvenes. Santiago. Ediciones Universidad Católica de Chile, 39-43, 73-77.

Gerris, J., Dekovic, M. y Janssens, J. (1997) The relationship Between Social Class and Childrearing Behaviors: Parents Perspective Taking and Value Orientations. Journal of Marriage and the Family, 59: 834-847.

Gómez, L. (1985) Dimensiones de Personalidad y Valores Interpersonales en Adolescentes. Revista Latinoaméricana de Psicología, 17, 2: 193-203.

Hoffman, Paris y Hall (1997) Psicología del Desarrollo Hoy. Vol. II, Madrid, España.Mc Graw Hill.

Kohlberg , L. (1987) Child, Psychology and Childhood Education. Longman Edit.

Levy, M. (1975) El Proceso de Modernización y la Estructura de la Sociedad. Una Perspectiva para el Análisis de los Asuntos Internacionales. Madrid, España. Editorial Aguilar, 301-346.

MANLove, J. (1997) Early Motherhood in an intergenerational perspective: The experience of a british cohort. Journal of Marriage and the Family 59, 2: 263-279.

Maddaleno, M. (1986) Enfoque familiar y los Problemas de Salud en el Adolescente, en Salud Familiar, Santiago, Chile. División Ciencia Médica Oriente, Facultad de Medicina, Universidad de Chile, 125145.

Mathiesen, M.E. , Mora, O. y Castro, M. (1999) Comportamiento Sexual de los Estudiantes Universitarios y Características familiares y personales asociadas. Avances en Psicología Clínica Latinoamericana. Bogotá Colombia.17: 7-20.
Mathiesen,M.E. y Mora, O. y Castro, M. (1998) Valores de los estudiantes de la Universidad de Concepción, Chile, una aplicación de la Escala de Comportamientos Morales Controvertidos, Revista Latinoamericana de Psicología, 30, 1: 121136.

Mathiesen, María Elena (1989) Modernidad y Funcionamiento Familiar en la provincia de Concepción, Revista de Sociología, Universidad de Chile. 4: 31-46,

McLnahan, S.S., y SAndefur, G (1994) Growing up with a single parent: What hurts, what helps. Cambridge, MA: Harvard University Press.

McLnaHan, S.S. (1988) Intergenerational consequences of family disruption .American Journal of Sociology, 94: 130-152.

Mestre Escriva, V.; Perez Delgado, E.; Samper García, P. (1999) Programas de Intervención en el Desarrollo Moral: Razonamiento y empatía. Revista Latinoamericana de Psicología, 31, 2: 251-270.

Merino, J.M. (1987) Independencia y Modernidad Psicosocial. Tesis para obtener el grado de Magister en Ciencias Sociales. Universidad de Chile, Santiago.

Montenegro, H. (1995) Familia y Sociedad: Una Relación en Crisis". Revista Trabajo Social, Pontificia Universidad Católica de Chile. 65: 17-27.

Navarro, G. , González A. y Recart, I. (2000) Participación de los padres en el proceso educativo de sus hijos: estudio exploratorio en dos escuelas municipales. PAIDEA, 28: 127-141.

Papalia, D. (1997) Desarrollo Humano. Bogotá, Colombia. Mc Graw Hill.

Rice, Phillip (1997) Desarrollo Humano. Ciudad de México, México. Prentice Hall Hispanoamericana. 
Robinson, J., SHAVER P., y WRightSMAN (1991) Measures of Personality and Social Psycological Attitudes. Vol 1, San Diego, California, Academic Press, 742-746.

Roberts y Feetham (1982) Assesing Family Functioning. Across three areas of relationship. Nursing Research, Philadephia, P.A. 31: $232-234$.

Tamayo, A. (1993) Valores de los Adolescentes: Ejes Motivacionales, Acta Psiquiátrica y Psicológica de América Latina. 39: 140-151.

VAlenZuela, M. E. (1995) Hogares con Jefatura Femenina, una realidad Invisible. Proposiciones, 26: 117-130.
Vigneaux, M. E. (1990) Adaptación de un Instrumento para evaluar el Desarrollo del Juicio Moral. Tesis para optar al título de psicólogo. Pontificia Universidad Católica de Chile.

Williams, Robin, J.R. (1979) Valores: Concepto de Valores. Enciclopedia Internacional de las Ciencias Sociales. Tomo X. Madrid, España. Editorial Aguilar, 610.

WU, L. y B. MARTISON (1993) Family structure and risk of marital birth. American Sociological Review, 58: 210-231.

Wu, L. (1996) Effects of family instability, income, and income instability on the risk of premarital birth. American Sociological Review, 61: 386-406.

Período de límite de recepción de artículos: 30 de Marzo 2002. Fecha de aceptación artículos: 30 de octubre 2002. 